go for several days, finally subside, and a recovery ensue, with no greater inconvenience than tenderness and slight irritation of the skin for a short time afterwards. The same causes that produce the eruption in a single member of a family, are likely to influence the remainder; and hence it may actually be epidemic in a family without its necessarily extending to other persons.

At the St. Pancras Royal Dispensary, a mother brought to me her child, two years and nine months old, covered over the head, the neck, and the face with a distinct and well-marked sudaminal eruption, the small vesicles being as large as millet seeds, surrounded by a red base, and accompanied by profuse sweating. This eruption had commenced six days before, and had disappeared and become renewed several times; and now isolated groups of the vesicles were appearing on other parts of the body, and in some places (as between all the fingers and on the back of the hands) solitary vesicles showed themselves. The child was cross and fractious, and no doubt suffered from the well-known pricking or tingling sensation so characteristic of miliaria. On looking from the child to the mother, she was found to be suffering from the same thing, but in a milder degree, the eruption being confined to the face and the flexures of the arms. Her other children, six in number, were likewise all, though variously, affected by it: thus, in some, the eruption was chiefly confined to the face, head, and neck; in others, to the limbs; and in one, it seemed to be associated with boils over the hip, rendering her for the time lame.

Here, then, was an entire family affected, except the father, which I attribute to the grest heat, and which had so oppressed all of them as to cause their being " overwhelmed with perspira. tion, actually dripping with it," as the mother described. The sudamina followed upon this, probably induced by copious draughts of water and other liquids taken to assuage the intense thirst consequent on the great drain upon the system by the sweating. Looking upon the disease here as the result of the profuse perspirations produced by the excessive heat on the skin, and not as arising from any gastro-intestinal disturb. ance, I prefer to adopt the name which most truly expresses its general meaning. I have seen violent exercise under great heat followed by the perspirations and the sudaminal eruption, which has lasted from two to three days-occasionally only twenty-four hours. If the weather continues warm and oppressive, the duration of the disease may extend to weeks; but the treatment which I have found most serviceable to prevent this, both here and abroad, is small doses of antimonials, nitrate of potass, and tincture of opium. The last is greatly serviceable in quieting the irriration; the first checks the perspiration, and the tendency to get rid of fluid is relieved by the kidneys.

The mother and the children greatly improved under the treatment pursued, the eruption dying off the head, face, and neck, but it was followed by scattered boils in various parts of the body. Before the mother got better, the eruption ex. tended along the anterior part of the arms to the hands, then appeared on the back of the arms, and finally upon the neck.

Portman-street, Portman-square, July, 1859.

\section{CASE ILLUSTRATIVE OF THE POST-MOR- TEM ACTION OF THE GASTRIC JUICE ON THE STOMACH AND DIAPHRAGM.}

Bx W. GRANTT, M.B., ASSISTANT-SCRGEON H.M. 31st REGIMENT, POONA.

Private Edward V-, of H.M. 3lst Regiment, aged thirty, has been in the service five years; was a shoemaker by trade before his enlistment; has a fair complexion; is a stout built, middle-sized man, of rather strumous appearance. $\mathrm{He}$ was admitted into the Regimental Hospital, Poona, on the 15th of March last, complaining of acute headache, with vertigo, nausea, heat of skin, thirst, \&c.; tongue foul; bowels irregular; pulse 96 . He was treated with aperients and quinine, and, in three days after was convalescing, when he had an attack of slight catarrhal ophthalmia from sleeping near an open window. From this affection he made a satisfactory re. covery, and was discharged for duty on the 24th of March.

On the morning of April 1st, he was re-admitted, complain- ing of headache, and seemingly much in the same state as on the 15th of March. Soon after admission, he had a severe rigor, accompanied with most acute throbbing pain in the right side of the head. He was ordered some calomel and James's powder, followed in three hours by a dose of compound powder of jalap, with sulphate of quinine. After the action of the purgative, he felt considerably relieved.

On the morning of the 2nd of April, he complained of shooting pain in the right eye and ear, and along the right side of the face, and tenderness on pressure over the scalp on the right side. Leeches were applied to the right temple, and, in the evening, he had a warm bath, foilowed by ten grains of Dover's powder, and a large blister behind the right ear.

Next day the pain had in a great measure subsided. The blister had acted well, and was ordered to be kept open. From this time up to the 13th he seemed to mend slowly. He had occasional pain in the head of a shooting character, but said he felt himself recovering. He had no chest nor abdominal symptoms of an untoward nature, and was able to eat some arrowroot, or beef-tea with a little bread, from time to time. $\mathrm{He}$ was taking two grains of iodide of potassium, with an ounce of infusion of chirretta, three or four times a day, and an occasional aperient.

On the evening of the 13th of April, he lapsed into a state of coma, and his pulse became slow and laboured. From this time he nover rallied, but died on the 15th of April, at seven A.M.

Sectio cadaveris five hours after death. - General appearance of the body: Iimbs and trunk well developed and muscular; marks of leeching and blistering on the right side of the head; no other cicatrices or marks on any part of the body. Head: On opening the skull, the dura mater was found adherent to several portions of the right hemisphere of the brain by processes of well-organized lymph. On removing the brain, an abscess, of the size of a hen's egg, containing thick pus, was found in its substance, just over the petrous portion of the right temporal bone, the subjacent part of which was in a state of caries. The lateral ventricles contained a quantity of grumous-looking serum; choroid plexus much congested. The rest of the brain seemed healthy. Chest: No pleuritic adhesions on either side; the left pleural cavity contained nearly a pint of blackish, fluid blood; the lungs had a few crude tubercles scattered throughont their substance; their posterior portions were engorged with blood; pericardium and heart healthy. The diaphragm presented a remarkable appearance; it contained a large ragged aperture, with dark edges immediately to the left of the spinal column, and, further to the left, a smaller aperture of a similar character. The stomach, which seemed lacerated, protruded into the thoracic cavity throngh the larger opening in the diaphragm. Abdomen: No traces of inflammation in the peritoneum; intestines much distended with flatus. Nearly the whole of the stomach, at its greater curvature, seemed to have been dissolved away by the post-mortem action of the gastric juice, and this process appeared to have extended to the diaphragm; the edges of the aperture in the stomach were of a blackish or deep ashgrey colour, soft, and very ragged. The vessels in some parts of the remaining mucous membrane presented a peculiar brownish, arborescent appearance. (There were no traces of inflammation in the mucous membrane of the cesophagus.) The liver, spleen, kidneys, and other abdominal viscera were perfectly healthy.

Remarks.-In the greater proportion of cases where extensive solution of the stomach and neighbouring parts by the post-mortem action of the gastric juice has been found to exist, the individual had died shortly after having taken food into the stomach; but in this instance, nothing in the shape of food, except an occasional teaspoonful of weak wine-and-water, had been taken after the evening of the $13 \mathrm{th}$, nearly thirty-six hours before death. It is probable that an excessive elimination of the gastric secretion was produced, through reflex action, by the diseased brain, as suggested by Dr. Budd. The chemical action would also take place more readily in a climate of high temperature, where the heat of the body is longer retained. The temperature in this case was $76^{\circ} \mathrm{Fahr}$. in the shade at the time of death, and $94^{\circ}$ when the examination of the body was made. The protrusion of the stomach into the thorax (which, according to Dr. Alfred Taylor, uniformly takes place in ex. tensive wounds of the diaphragm during life) was most probably produced by the gaseous distension of the intestines. The blood found in the left pleural cavity must have oozed from the severed edges of the stomach and diaphragm.

Bombar, May, 1859 